\title{
Noisy Radial-blurred Images Restoration Using the BM3D Frames
}

\author{
Li Chonglun*, Liu Zhong and Yang Lujing
}

College of Electronic Engineering, Naval University of Engineering, Shenyang, Wuhan, 430033, P.R. China

\begin{abstract}
BM3D frames based formulation provides new perspectives for the use of BM3D modeling in the circular shift-invariant reconstruction, but cannot be applied in the non-variant reconstruction. In order to solve this problem, a radial blur physical model was established, in this paper rectangular block replaced sector block in rectangular coordinate image, the matching area and restoration coefficient regulation were established to improve the BM3D frames and to complete the radial image restoration. The results show that the sector block was matching in neighbor area, the radial blur image can be effectively recovered, and the effect is obviously better than RL-NL and RL-TV methods or other restoration methods.
\end{abstract}

Keywords: BM3D frames, image restoration, radial blur.

\section{INTRODUCTION}

Image restoration is a key step in the field of image processing [1]. This technology is used in solving the blurry and noisy images restoration problem in the space environment in the image observing area and the motion platform imaging observing area. Nowadays, the Aerial Videography technology has been widely used and the motion speed of observation platform has been improved a lot, which has an effect on the image blurry phenomenon. It mainly reflected in high speed motion of load platform shift invariance with radial blur. So this research on how to improve the recovering effect of the radial blurred image with noise has important practical significance.

At present there are a lot of related researches on the noisy blur image restoration problem. Those methods are usually originated from various methods such as probability theory, statistics theory, linear and nonlinear filtering theory, etc. And the RL (Lucy - Richardson) algorithm is one of the most well-known $[2,3]$ methods, which is based on the bayesian estimation theory, using the iterative way to estimate the actual fuzzy kernel function and the real image. This method is used in the non-noisy blur image restoration, without considering the noise influence in the process of recovery, so that it cannot meet the actual demand in the treatment of the blurred image with noise.

In order to prevent the noise influencing recovery effects, the researchers had considered introducing the simple filtering denoising algorithm with the form of regularization item into the recovery process, in $2003 \mathrm{DE}$ monvel JB et al. introduced Tikhonov regularization method into RL algorithms, with the assumption that the original image was smooth. This method could eliminate the influence of noise to a certain extent, but because of its assumption, the restored images are prone to leading parasitic ripple edge fuzzy phenomenon [4]. After that, Dey et al. took the ROF (TV) algorithm [5] proposed by Osher in 1992 as a total variation regularization item and introduced it into the RL algorithms, which was named as RL-TV algorithm then, and obtained a good effect [6].

In 2007, the NL denoising method showed up, using the image of redundant information as an important means of image denoising. In 2009, YiFei Lou et al. introduced NL method into RL algorithms [7]. In 2011, through the analysis and synthesis methods, Aram et al. transferred fuzzy image restoration problems with noise into to the associated fuzzy and denoising problems $[8,9]$, made $\mathrm{BM} 3 \mathrm{D}$ and other denoising methods can be directly used in noise image fuzzy, and achieved obvious effects. But the current recovery methods are mostly based on the assumption of blurred images having moved the same nature, so it is difficult to effectively recover signals with noise for radial blur this shift becomes blurred image.

In order to solve this problem, this paper attempts to establish the model by analyzing the physical process of radial blur form through the transformation of BM3D image matching template, to delimit the scope of template matching in the way of polar coordinates transformation to radial blur image restoration problem into BM3D framework for processing, and to improve the effect of image restoration by applying the BM3D algorithm to image using redundant information.

\section{PHYSICAL MODEL OF RADIAL BLUR}

The image restoration is a technology that changes the observed degradation images to the estimate images and makes those estimate images maximumly approximate the real images. Image degradation is mainly divided into two categories: (1) the blur (space degradation), caused by the relative motion between the photoelectric payload and target, such as flow disturbance, load out-of-focus factors (2) the 


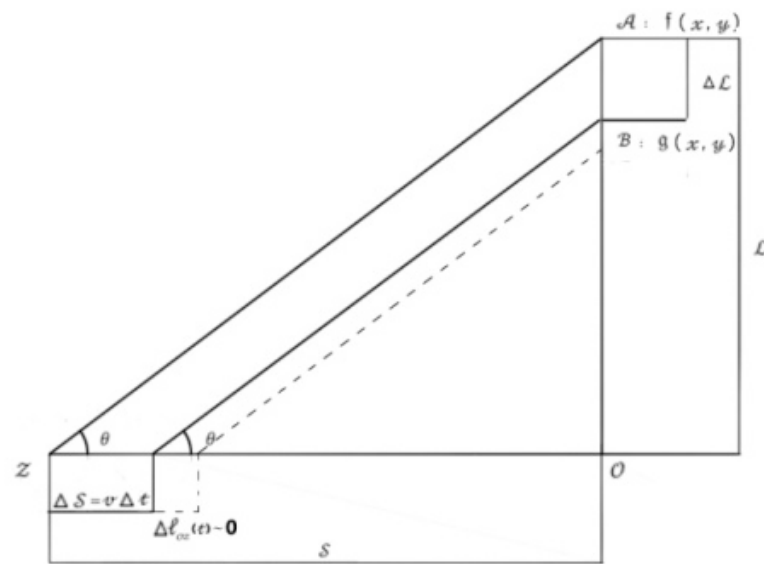

(a)

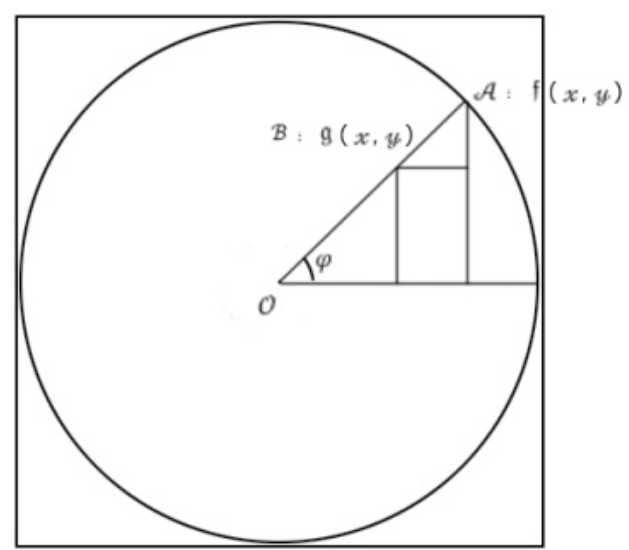

(b)

Fig. (1). Geometry relationship of motion plane and target plane.

noise (point degradation), caused by the interferences to the imaging components from electrostatic interference and other factors. The degraded image restoration process, even if the inverse process to estimate the original image, so get the right image degradation model is the key step in the restored image.

We consider the radial blur model and the general blur model have the same structure. Blur operator and additive zero-mean white Gaussian the conventional observation model is expressed as:

$g(x, y)=H(x, y) * f(x, y)+n(x, y)$

Where $f$ is the original image $g$ is observed data $H$ is a continuous linear operator $n$ is white noise. This restoration problem is an image deconvolution, it is the estimation of $f$ given the data $g$ and continuous operator $H$.

The radial blur image is generated by the relative high speed motion between platform and target plane in exposure time. So this model will be divided into motion plane (yoz plane and target plane (xoy plane)to analyze respectively [10].

The (Fig. 1) shows a motion plane. Platform moving along $\mathrm{oz}$ axis to point $\mathrm{o}$, movement speed is $v$, in $\Delta t,(\Delta t<T)$, the distance of platform movement is $\Delta S=v \Delta t$, shrink distance can be obtained by trigonometric function formula

$\Delta L=v \Delta t \times \frac{L}{S}=\zeta \cdot L$

In the target plane, a pure radial path is vector $A B$ in Fig. 1. (b):

$$
\begin{aligned}
g(x, y)= & \frac{1}{t} \int_{0}^{t} f(x+\Delta x, y+\Delta y) \\
& =\frac{1}{t} \int_{0}^{t} f(x+v t L(x, y) \sin (\phi(x, y)) / S, y-v t L(x, y) \cos (\phi(x, y)) / S) d t \\
& =\frac{1}{\Delta L(x, y)} \int_{0}^{\Delta L(x, y)} f(x+\Delta l \sin \phi, y-\Delta l \cos \phi) d s
\end{aligned}
$$

\section{BM3D FRAME}

BM3D model is the best model for noisy image. This model based on nonlocal (NL) method that get image denoising with adaptive and high order groupwise. This model proposed by Dabov et al., is divided into two similar steps. In this paper combining the definitions from Reference [9] and Reference [11], we describe it as three segments.

Let the image $f$ can be represented as a $\sqrt{N} \times \sqrt{N}$ square matrix, and each image block is $N_{b l} \times N_{b l}$, it can be square represented to $\sqrt{N_{b l}} \times \sqrt{N_{b l}}$, denote a vector of elements of $j$-th block $F_{j}$ by $f_{j}$ and define $P_{j}$ as an $N_{b l} \times N$ matrix of indicators $[0,1]$. So that $f_{j}=P_{j} \cdot f$.

\subsection{Analysis}

Divide image into equal to the size of the blocks based on equal step size, the distance regulation is built for the 3-D group that is:

$$
\begin{aligned}
& S_{x R}^{h t}=\left\{x \in X: d\left(f_{x R}, f_{x}\right) \leq r_{\text {match }}^{h t}\right\} \\
& d\left(f_{x R}, f_{x}\right)=\frac{\left\|\gamma^{\prime}\left(\tau_{2 D}^{h t}\left(f_{x R}\right)\right)-\gamma^{\prime}\left(\tau_{2 D}^{h t}\left(f_{x}\right)\right)\right\|_{2}^{2}}{\left(N_{1}^{h t}\right)^{2}}
\end{aligned}
$$

where $r_{\text {match }}^{h t}$ is the maximum distance for which two blocks are considered similar. $\gamma^{\prime}$ is hard-thresholding operator with threshold $\lambda_{2 D \sigma}$ and $\tau_{2 D}^{h t}$ denotes the normalized 2D linear transform.

Denote the maximum size of group by setting an upper bound $K, r$ is the number of group, $J$ is set of group, so that $J_{r}=\left\{j_{r, 1}, \ldots, j_{r, K}\right\}$, and $J=\left\{J_{r}: r=1, \ldots, R\right\}$, where $R$ is the maximum number of groups.

\subsection{Processing}

Denote $D_{2}$ is $\sqrt{N_{b l}} \times \sqrt{N_{b l}}$ size representing respectively 1-D interblock transforms and $D_{1}$ is $K \times K$ size representing respectively $1-\mathrm{D}$ intrablock transforms. The separable 2-D 
transforms for the block $F_{j}$ is $\Theta_{j}=D_{2} F_{j} D_{2}^{T}$. The vectorization of this formula using the Kronecker matrix is $\theta_{j}=\left(D_{2} \otimes D_{2}\right) \cdot f_{j}$, where $\theta_{j}$ and $f_{j} \in R^{N_{b l}}$ corresponding to the matrices $\Theta_{j}, F_{j}$.

So this 3-D group is $\left\lfloor\theta_{j_{r}, 1}, \ldots, \theta_{j_{r}, K}\right\rfloor$, using the 1-D interblock transform to every row of the matrix is $\Omega_{r}=\left\lfloor\theta_{j_{r}, 1}, \ldots, \theta_{j_{r}, K}\right\rfloor D_{1}^{T}$. Finally we express the 3-D group spectrum coefficients as a formula which is:

$\omega_{r}=\sum_{j \in J_{r}}\left(d_{j} \otimes\left[\left(D_{2} \otimes D_{2}\right) \cdot P_{j}\right]\right) f$

where $d_{j}$ is $J$-th column of $D_{1}$

Finally the formula is:

$\phi_{r}=\sum_{j \in J_{r}}\left(d_{j} \otimes\left[\left(D_{2} \otimes D_{2}\right) \cdot P_{j}\right]\right)$

$\omega=\left[\begin{array}{c}\phi_{1} \\ \vdots \\ \phi_{R}\end{array}\right] \cdot f=\phi f$

\subsection{Synthesis}

After completing the processing segment, the filtered providing estimates for each $N \times N_{b l}$ size block matrix in the group through the inverse 3-D transform $\psi_{r}=\sum_{j \in J_{r}} d_{j}^{T} \otimes\left[P_{j}^{T}\left(D_{2} \otimes D_{2}\right)^{T}\right]$, this block estimate is returned to their original position. The final image estimate is defined as the weighted mean of the group estimates. So this synthesis operator is:

$f=\psi \omega=W^{-1} \cdot\left[g_{1} \psi_{1}, \ldots, g_{R} \psi_{R}\right] \cdot \omega$

where $W=\sum_{r} g_{r} \sum_{j \in J_{r}} P_{j}^{T} P_{j}$

In order to deal with the noisy-blur image reconstruction problem, traditional frame usually allows two different formulations depending on which kind of image modeling is, the Reference [11] provides a frame, and this frame is a combination of deblur and denoise model, based on the Nash equilibrium balance of two object function.

The deblurring problem is like the following constrained optimization:

$$
\begin{aligned}
& f^{*}=\arg \min _{f} \frac{1}{2 \sigma^{2}}\|g-H f\|_{2}^{2} \text { subject to }\left\|f-\psi \omega^{*}\right\|_{2}^{2} \leq \varepsilon_{1} \\
& \omega^{*}=\mathfrak{I} \eta_{\tau \omega}\left(\phi f_{t+1}\right)
\end{aligned}
$$

So this iterative decoupled deblurring BM3D algorithm is:

$$
\begin{aligned}
& f_{t+1}=\left[\frac{1}{\sigma^{2}} H^{T} H+\frac{1}{\gamma} I\right]^{-1} \times\left[\frac{1}{\sigma^{2}} H^{T} g+\frac{1}{\gamma} \psi \omega_{t}\right] \\
& \omega_{t+1}=\mathfrak{I} \eta_{\tau \omega}\left(\phi f_{t+1}\right)
\end{aligned}
$$

\section{MATCHING AREA AND RANGE}

Although the reconstruction effect of BM3D frame is better than other methods, this method can only be applied to shift invariant image processing [11]. According to the analysis for the physical model of radial blur in the section 1, the radial blur image model is a shift variant model. So the BM3D frame cannot be directly used to reconstructing the radial blur image. In order to apply BM3D frame to the radial blur image, the key is to change the shape of reference block to build the shift invariant nature in a limited range.

According to the characteristics of the radial blur, each point in the orginal noisy image is the mean of all points in radial path. There is equal radial distance from equal radius in noisy radial blur image, so each rows element has equal feature in polar coordinates system image, so the BM3D frame can be used in limited equal radius of radial blur image in polar coordinates system.

In this paper, denote the sector block instead of the rectangle block in rectangular coordinate system (Fig. 2). show the sector block:

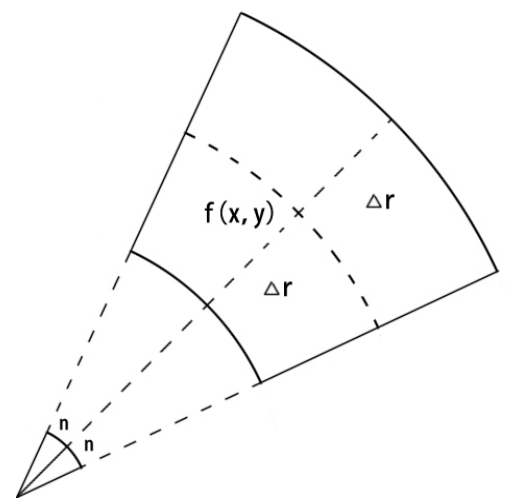

Fig. (2). Sector Block.

The sector-shaped formula is:

$S_{b l}=\frac{n \pi r_{\max }^{2}}{360}-\frac{n \pi r_{\min }^{2}}{360}=\frac{n \pi}{180} \Delta r \cdot r_{\text {mid }}$

Based on the features of sector block, each point of the block corresponding to the other one in equal radius, so the matching scope is limited in equal radius. But this block show the superposition of all blocks in radial path, if only matching in single radius, the enough similar blocks can't be found and the effect of filter will be reduced. In order to ensure the BM3D frame's efficiency, we expand the matching range in neighbor radius. So it needs to make equal area in all block to ensure the consistency of information between the blocks in different radius, that is:

$S_{1}=S_{2} \Rightarrow \frac{n_{1} \pi}{180} \Delta r_{1} \cdot r_{\text {mid }, 1}=\frac{n_{2} \pi}{180} \Delta r_{2} \cdot r_{\text {mid }, 2}$

Based on Curvature formula, top and down radian is different in variously $r_{\text {mid }}$ when it has equal $\Delta r$. It shows the direct relationship between the shape of block and the $r_{\text {mid }}$. So we change the weight define for different block in different position is $g_{r}^{\prime}=\mu \cdot g_{r}$, where $\mu=\frac{\left|r_{\text {mid }, i}-r_{\text {mid }, j}\right|}{r_{\Delta \text { mid }}}$. 


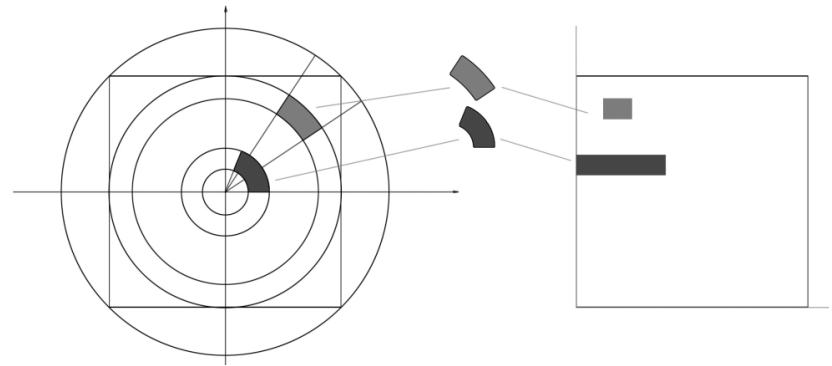

Fig. (3). Matching Block in Rectangular Coordinate System and Polar Coordinate System.

The sector block in rectangular coordinate system will change to the rectangle in the polar coordinate system (Fig. 3). shows this problem:

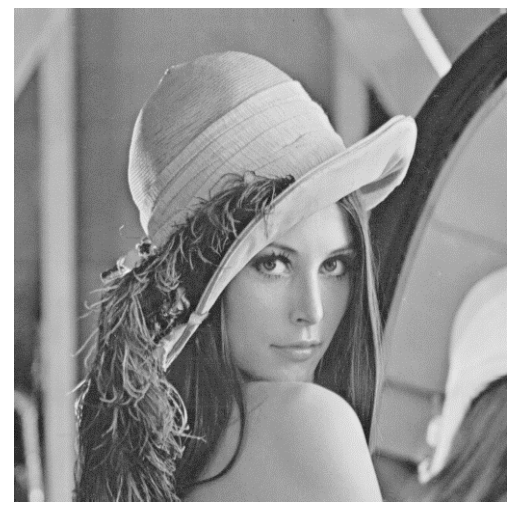

Origin image

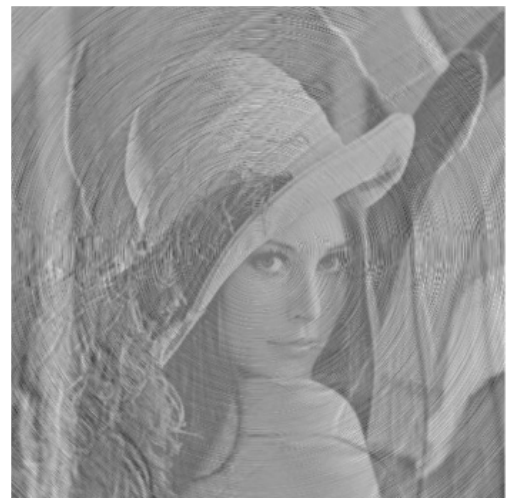

RL

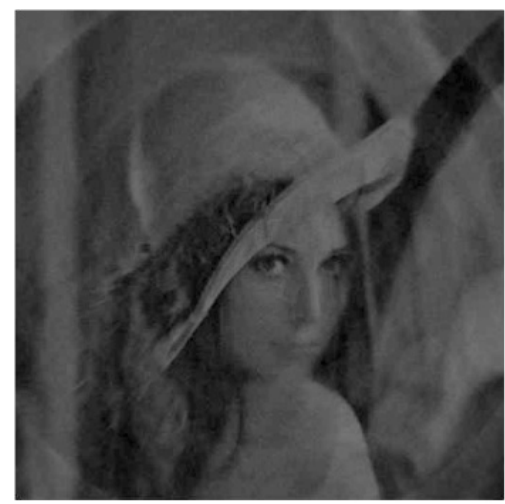

RL_NL
The (Fig. 3). shows, the angle of sector block proportional size of block in polar coordinate system. So the block will change the size to the reference block, then error is generated in this operator. In order to solve this problem, the flexible parameter $\mu$ is added to weight is $g_{r}^{\prime}=\mu^{2} \cdot g_{r}$.

The synthesis formula is

$$
\begin{aligned}
& f=\psi \omega=W^{-1} \cdot\left[\mu_{1}^{2} g_{1} \psi_{1}, \ldots, \mu_{R}^{2} g_{R} \psi_{R}\right] \cdot \omega \\
& W=\sum_{r} \mu_{r, j} g_{r} \sum_{j \in J_{r}} \mu_{r, j} P_{j}^{T} P_{j}=\sum_{r} g_{r}^{\prime} \sum_{j \in J_{r}} \mu_{r, j} P_{j}^{T} P_{j}
\end{aligned}
$$

In this paper, denote the matching range is:

$$
r_{\text {mid }}-\frac{\Delta r}{2} \leq r_{\text {mid }} \leq r_{\text {mid }}+\frac{\Delta r}{2}
$$

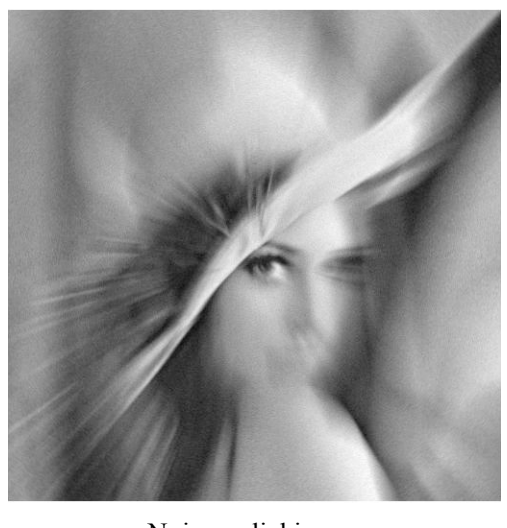

Noisy-radial image

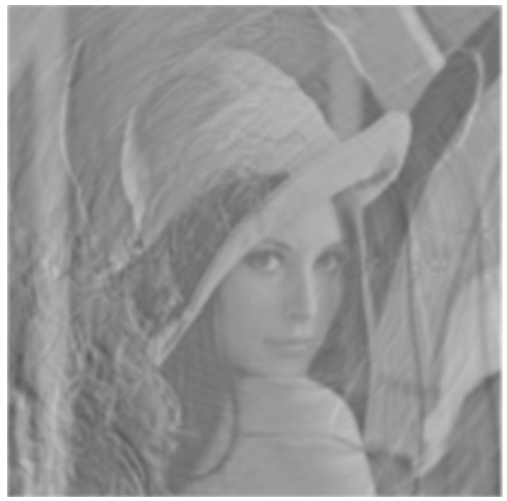

RL_TV

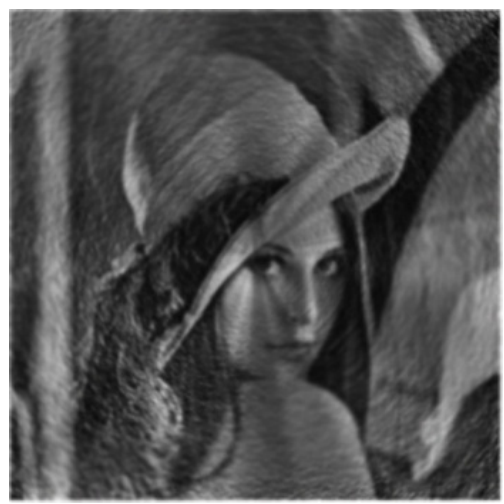

BM3D

Fig. (4). Result of Restoration (Lena, $\zeta=0.3$ ). 


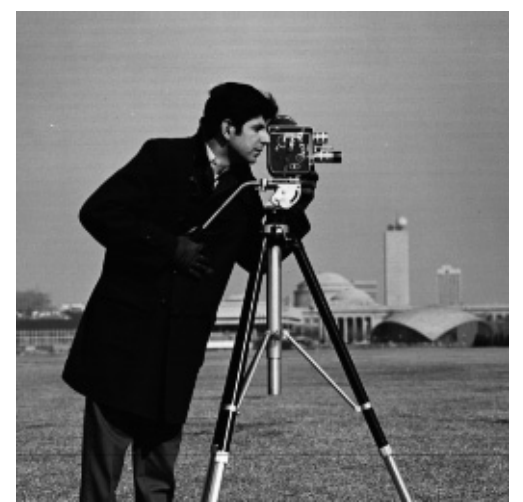

Origin image

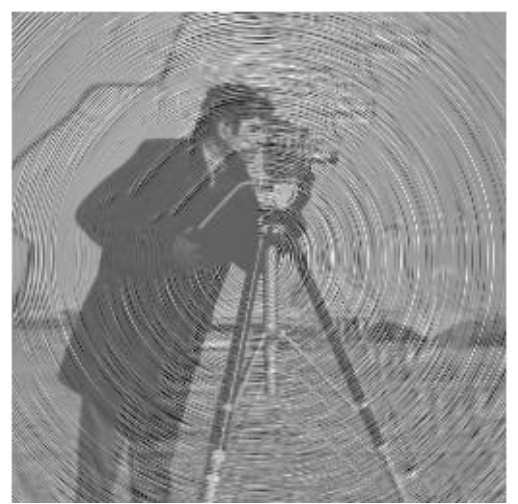

RL

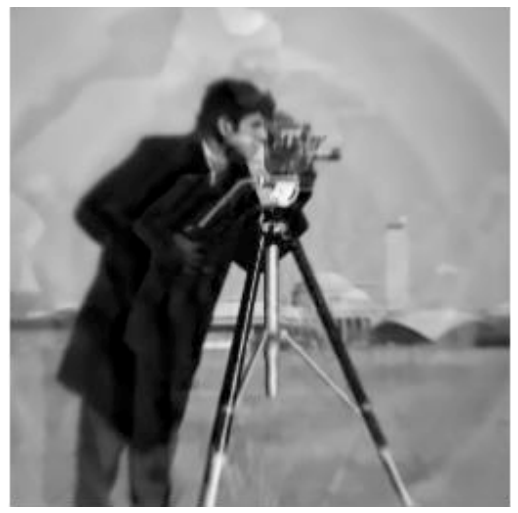

RL_NL

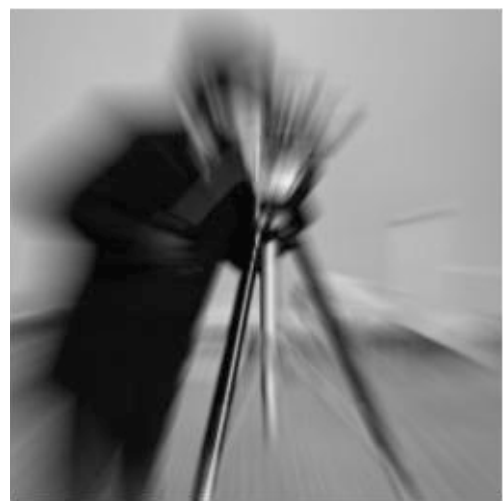

Noisy-radial image

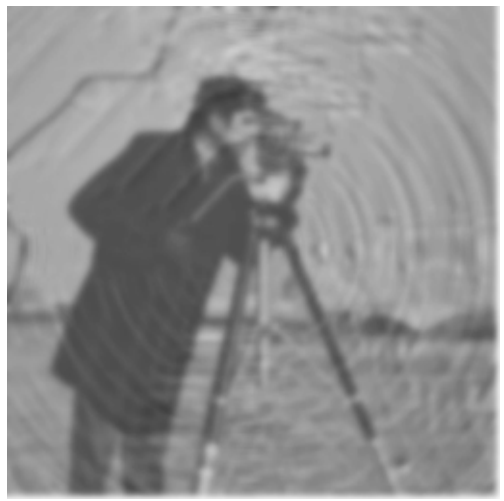

RL_TV

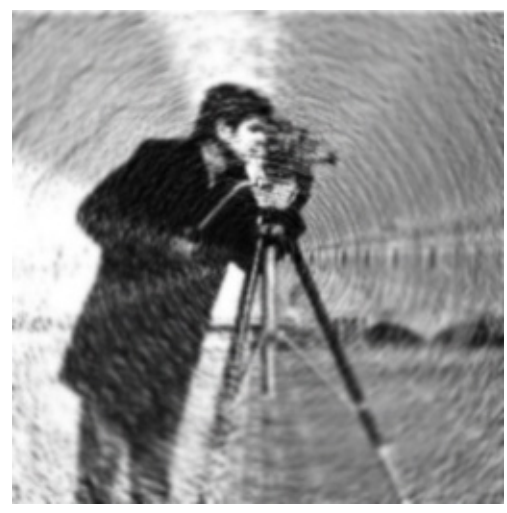

BM3D

Fig. (5). Result of Restoration (Cameraman, $\boldsymbol{\varsigma}=0.3$ ).

\section{EXPERIMENT AND ANALYSIS STUDY}

The test environment is matlab2014 in Dual-Core CPU, memory is $4 \mathrm{G}$ computer. It compares the RL, RL-TV, RLNL methods with the improved BM3D frame method by using the PSNR and MSSIM evaluation function.

In this test, radial blur parameter is $\varsigma=0.3$ image in radial blur restoration, additive zero-mean and variance is $\delta=10^{-3}$ white Gaussian noise, construction a restoration error parameter is $5 \%$. Regularization parameter is 0.8 in RL-TV method, and the parameter of RL-NL method is from the reference [12]. Reference image is Lena and Cameraman (Figs. 4 and 5). show the result of restoration, and (Table 1) show result of evaluation.
Table 1, (Figs. 4 and 5). shows the effect of the improved-BM3D frame is better than RL-NL (in $\varsigma=0.1,0.2$ ), RL-TV and TV method, but it is poorer than RL-NL (in $\varsigma$ $=0.3$ ); each effect of restoration methods is reduced with improving the parameter $\zeta$, the RL method can restore the radial blur but produce a biggest ringing-effect, the ringingeffect is limited smoothed by using the RL-TV method and keep the main edge.

The improved-BM3D frame is similar to RL-NL method. Comparing these two methods alone, the (Fig. 6) shows the details from these two methods in Lena and Cameraman images. This test shows the detail of improved-BM3D is better than the RL-NL in 'Lena' and poorer in 'Cameraman'. 
Table 1. Result of Restoration Coefficient (Cameraman).

\begin{tabular}{|c|c|c|c|c|c|c|}
\hline \multirow{3}{*}{$\boldsymbol{S}$} & Evaluation Function & Blur Image & RL & RL-TV & RL-NL & BM3D Frame \\
\hline \hline \multirow{2}{*}{0.1} & PSNR & 20.042 & 22.916 & 23.001 & 28.043 & 28.131 \\
\cline { 2 - 7 } & MSSIM & 0.4212 & 0.7033 & 0.7196 & 0.8245 & 25.783 \\
\hline \multirow{2}{*}{0.2} & PSNR & 17.290 & 19.528 & 19.702 & 0.7360 \\
\cline { 2 - 7 } & MSSIM & 0.4087 & 0.6013 & 0.6428 & 25.833 \\
\cline { 2 - 7 } & PSNR & 15.605 & 16.994 & 17.013 & 24.707 & 24.761 \\
\hline
\end{tabular}

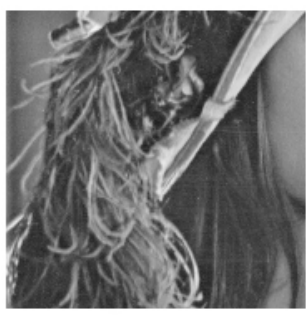

Lena

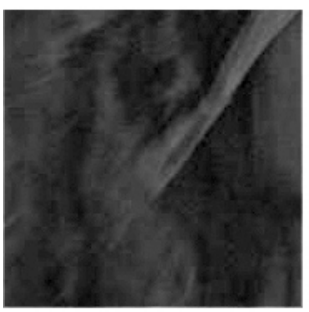

RL-TV

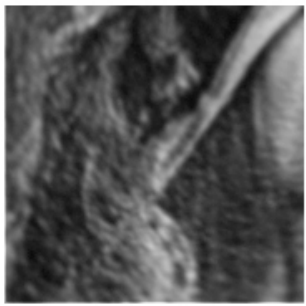

BM3D

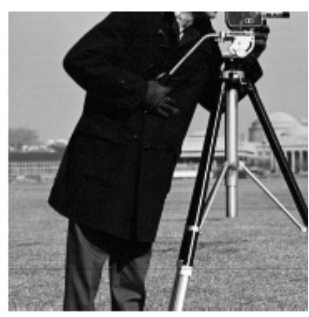

Cameraman

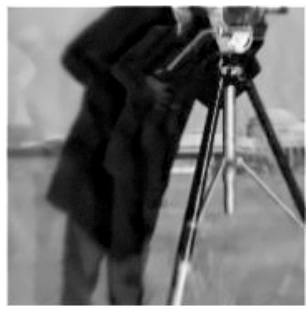

RL-TV

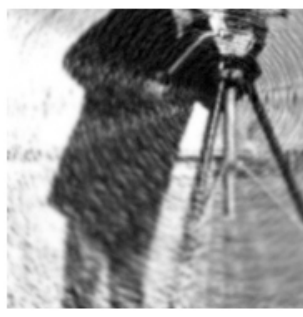

BM3D
Fig. (6). Result of Restoration (Cameraman, $\varsigma=0.3$ ).

Lena shows a large number of details and 'Cameraman' is more smoothly than 'Lena', it shows the influence of ringing-effect is reduced from complex details in iteration operator, so this improved-BM3D method is better than other method in complex image.

\section{CONCLUSION}

In this paper, the noisy radial blur physical model is set up. According to the features of radial blur image, it ensures a matching range, changes the rectangle block to the sector block in BM3D frame, improves the weight function for synthesis step, and compares the RL, RL-TV, RL-NL methods with the improved BM3D frame method by using the PSNR and MSSIM evaluation function. The result of test shows the improved-BM3D frame is better than other method when the $\varsigma=0.1$ or $\zeta=0.2$, then the speed of improved-BM3D frame is slower than other method.

\section{CONFLICT OF INTEREST}

The authors confirm that this article content has no conflict of interest.

\section{ACKNOWLEDGEMENTS}

This work was financially supported by the advance research fund of national ministries (9140A01060113JB1101).

\section{ABOUT THE AUTHORS}

First Author Li Chonglu, master degree studying for PhDs in Naval University of Engineering, The author's major is Image Recognition. 4 papers received by EI or SCI and 1 patents had been published.

Second Author Liu Zhong, Naval University of Engineering, Professor. The author's major is Target Tracking.

Third Author Yang Lujing, Naval University of Engineering, Professor. The author's major is Target Tracking.

\section{REFERENCES}

[1] H. Shen, S. Li, J. Mao, "Digital Image Restoration Techniques A Review", J. Image Graph., vol.14, no. 9, pp.1764-1775, 2009.

[2] W. H. Richardson, "Bayesian-Based Iterative Method of Image Restoration", J. Opt. Soc. Am., vol. 62, no. 1, pp. 55-59, 1972.

[3] L. B. Lucy, "An Iterative Technique for the Rectification of Observed Distribution", Astron. J., vol. 79, no. 6, pp.745-754, 1974.

[4] J. Da Rugna, H. Konik,"Automatic blur detection for meta-data extraction in content-based retrieval context", in International Society for Optical Engineering 2004, vol 5304, pp. 285-294, 2003.

[5] L. I. Rudin, S. Osher, E. Fatemi, "Nonlinear total variation based noise removal algorithms", Physica D: Nonlinear Phenom., vol. 60, no. 1, pp.259-268, 1992.

[6] Y. W. Tai, P. Tan, M. S. Brown, "Richardson-lucy deblurring for scenes under a projective motion path", Pattern Analysis and Machine Intelligence, IEEE Trans., vol. 33, no. 8, pp.1603-1618, 2011.

[7] Y. Lou, X. Zhang, S, Osher, H. Xu and Y. Liu, "Image recovery via nonlocal operators", J. Sci. Comput., vol. 42, pp. 185-197, 2010.

[8] A. Danielyan, M. Vehvilanen, A. Foi, V. Katkovnik, K. Egiazarian, "Cross-Color BM3D Filtering of Noisy Raw Data", J. Sci. Comput., vol. 233, pp. 2279-2283, 2009. 
[9] A. Danielyan, V. Katkovnik, K. Egiazarian, " BM3D frames and variational image deblurring", Image Processing, IEEE Trans., vol. 21, no. 4, pp.1715-1728, 2012.

[10] Y. Yang, M. Gao, J. He, "Research on Recovery Technology of Noisy Radial-Blurred Images", Machinery Des. Manufac., vol.7, pp. $98-100,2013$.
[11] K. Dabov, A. Foi, V. Katkovnik "Image denoising ostadinby sparse 3D transform-domain collaborative filtering", Image Processing, IEEE Trans., vol. 16, no. 8, pp. 2080-2095, 2007.

[12] W. Liu, C. Wu, Q. Lv, "Research onrestoration algorithm of noisy radial-blurred images", Wuhan Ligong Daxue Xuebao(Journal of Wuhan University of Technology), vol. 33, no. 4, pp. 139-143, 2011 .

Received: September 22, 2014

Revised: November 30, 2014

Accepted: December 02, 2014

(C) Chonglun et al.; Licensee Bentham Open.

This is an open access article licensed under the terms of the Creative Commons Attribution Non-Commercial License (http://creativecommons.org/licenses/by-nc/3.0/) which permits unrestricted, non-commercial use, distribution and reproduction in any medium, provided the work is properly cited. 